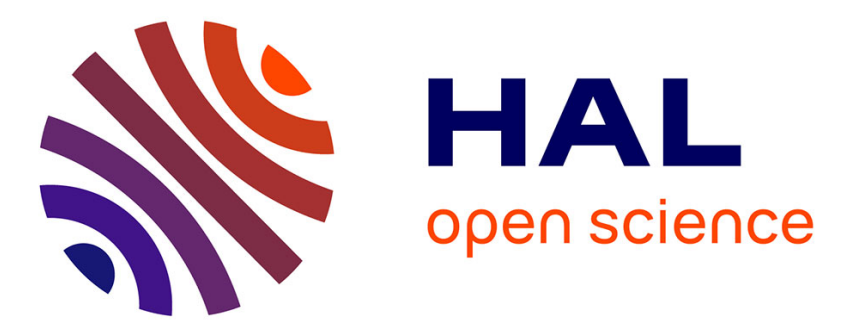

\title{
PID Switching Control for a Highway Estimation and Tracking Applied on a Convertible Mini-UAV
}

Gerardo Ramon Flores, Luis-Rodolfo Garcia Carrillo, Guillaume Sanahuja, Rogelio Lozano

\section{- To cite this version:}

Gerardo Ramon Flores, Luis-Rodolfo Garcia Carrillo, Guillaume Sanahuja, Rogelio Lozano. PID Switching Control for a Highway Estimation and Tracking Applied on a Convertible Mini-UAV. 51st IEEE Conference on Decision and Control (CDC 2012), Dec 2012, Maui, HI, United States. pp.31103115. hal-00776104

\author{
HAL Id: hal-00776104 \\ https://hal.science/hal-00776104
}

Submitted on 15 Jan 2013

HAL is a multi-disciplinary open access archive for the deposit and dissemination of scientific research documents, whether they are published or not. The documents may come from teaching and research institutions in France or abroad, or from public or private research centers.
L'archive ouverte pluridisciplinaire HAL, est destinée au dépôt et à la diffusion de documents scientifiques de niveau recherche, publiés ou non, émanant des établissements d'enseignement et de recherche français ou étrangers, des laboratoires publics ou privés. 


\title{
PID Switching Control for a Highway Estimation and Tracking Applied on a Convertible Mini-UAV
}

\author{
Gerardo Flores $^{\star}$, L.R García Carrillo ${ }^{\dagger}$, G. Sanahuja ${ }^{\star}$, and R. Lozano ${ }^{\ddagger}$
}

\begin{abstract}
This paper reports current work on development and navigation control of an experimental prototype of a new tilt-rotor convertible aircraft (Quad-plane Mini Unmanned Aerial Vehicle). The goal of the work consists of estimating and tracking a road using a vision system, without any previous knowledge of the road, as well as developing an efficient controller for treat with situations when the road is not detected by the vision sensor. For dealing with this situation, we propose a switching control strategy applied in two operational regions: road detected and no road detected. The exponential stability is proved for the subsystem formed by the lateral position taking into account the switching boundaries between the operational regions. The control law is validated in the proposed platform, showing the expected behavior during autonomous navigation.
\end{abstract}

\section{INTRODUCTION}

Motivated by the theory of switching systems and the need of developing effective Mini UAVs controllers and state estimators, this research work deals not only with the attitude and position stabilization problems, but also with performing a path following mission. The goal is getting the Quadplane [1] to navigate autonomously and to follow the path represented by a highway model (formed not only by lines but also by smooth curves) with the aim of tracking ground vehicles, exploring areas and monitoring roads as well as highways. Once the Quad-plane has achieved its goal, it can proceed to another distant target performing a transition flight, from helicopter mode to airplane mode, and thereby cover vast areas. In this work, we will focus on the hover mode of the Quad-plane.

The road to be tracked is considered unknown, thus the vehicle must estimate the path in real-time. Furthermore, the mission must be performed in presence of external disturbances, which are present in the translational dynamics as well as in the attitude dynamics of the vehicle. Previous research works [2] have shown that the PD controllers work efficiently to stabilizing the attitude dynamics of the Quad-rotor. Moreover, the PD controllers have proved to be effective for navigation problems in Quad-rotors [3]. The aforementioned works were no dealing with external disturbance problems causing that the vision system losses

This work was partially supported by the Institute for Science \& Technology of Mexico City (ICyTDF) and the Mexican National Council for Science and Technology (CONACYT).

* are with the Heudiasyc UMR 6599 Laboratory, University of Technology of Compiègne, France. Gerardo Flores (gfloresc@hds.utc.fr), G. Sanahuja (gsanahuj@hds.utc.fr)

$\dagger$ is with the Electrical and Computer Engineering Dept. (ECE) of the University of California, Santa Barbara (UCSB) (lrgc@ece.ucsb.edu)

$\ddagger$ is with the Heudiasyc UMR 6599 Laboratory, UTC CNRS France and LAFMIA UMI 3175, Cinvestav, México. (rlozano@hds.utc.fr) the artificial landmarks, which provides relative position and velocity of the vehicle. A switching strategy for estimating the states of an airplane equipped with imaging, inertial and air data sensing systems is introduced in [4]. The proposed methodology demonstrates being capable of dealing with instants of time when the visual detection of the landmark fails. A proposed solution to handle the situation when the image is lost, is to implement different controllers, which is appropriate since the use of a PD controller in the position dynamics is not enough for attenuating disturbances (e.g. winds gusts [5]). The analysis and control of a VTOL aircraft at simulation level is shown in [6]. Here the authors demonstrate the stability of the complete system divided in three different modes using a common Lyapunov function.

A pointing downwards camera allows to estimate the vehicle's heading angle with respect to the road's longitudinal orientation, as well as the lateral distance that must be corrected in order to navigate exactly over the road. Two operational regions are defined with the purpose of developing a switching control strategy for estimation and tracking: the first one concerns the situation when the road is detected, while the second one deals when it is not. Inside these two regions, it is demonstrated that the system is stable, in addition the system is proved to be stable in the time when the switch between both regions occurs. This control was tested in real-time experiments on the Quad-plane platform, showing the expected behavior during the navigation task. It is important to say, that all the vision and control algorithms are embedded on the Quad-plane.

The body of the papers is as follows. In section II we present the problem statement. Next, in section III the model of the vehicle in hover mode is presented. After that, the methods for estimating the states of the Quad-plane are shown in Section IV. Section V presents a switching control strategy as well as the stability proof. Section VI presents the Quad-plane experimental platform. The behavior of the Quad-plane during real-time experiments is shown in Section VII. Finally, some conclusions and future works are presented in section VIII.

\section{Problem Statement}

The road following procedure can be detailed as follows. First, the vehicle performs an autonomous take-off, reaching a desired altitude $z_{d}$ over the road. Then, the heading of the vehicle $\psi$, is controlled to obtain a parallel positioning between the Quad-plane $x$-axis $x_{h}$ and the longitudinal direction of the highway $x_{r}$ (Fig. 3). The Quad-plane forward velocity $\dot{x}$ is maintained constant while the distance between 


\section{A. Relative $\psi$ heading angle computation}

The angle between the camera's $x_{c}$ axis and the point $\left(x_{F}, y_{F}\right)$ can be computed as $\psi_{r}=$ $\arctan \left(y_{F}-y_{I}, x_{F}-x_{I}\right)$, this value is used for obtaining the heading angle $\psi_{d}$ that will align the vehicle's longitudinal $x$-axis $\left(x_{h}\right)$ with the road's longitudinal axis $\left(x_{r}\right)$, (Fig. 3). Finally we can express $\psi_{d}$ as

$$
\psi_{d}=\psi_{r}+\frac{\pi}{2}
$$

the therm $\frac{\pi}{2}$ is added to adjust $\psi_{d}$ to zero when $\psi_{r}$ is vertically aligned with $x_{h}$.

\section{B. Lateral position with respect to the road}

Lets consider an image-based distance $e_{x}^{c}$ along the camera's $x_{c}$ axis, which is defined between the road's center of gravity projection $\left(x_{g}, y_{g}\right)$ and the vehicle's center of gravity projection $\left(x_{0}, y_{0}\right)$, (Fig. 3). If $x_{i n}>x_{f i}$, then $e_{x}^{c}=\left(\frac{x_{I}-x_{F}}{2}+x_{F}\right)-\frac{c_{w}}{2}$, where $c_{w}$ represents the image's width in pixels. If $x_{I}<x_{F}, x_{I}$ must be replaced by $x_{F}$ and vice-versa. Using $e_{x}^{c}$, the lateral position of the aerial vehicle relative to the road is estimated as

$$
e_{y}=z \frac{e_{x}^{c}}{\alpha_{x}}
$$

where $z$ represents the Quad-plane's height and $\alpha_{x}$ is the focal length of the camera, in the $x_{c}$ direction.

\section{Translational velocities estimation}

By implementing an optical flow algorithm to the camera's image, translational velocities can be estimated. Consider that the arrangement camera-vehicle is moving in a 3-D space w.r.t. a rigid scene. When performing an optical flow computation, the coordinates of the $\left(x_{i}, y_{i}\right)$ features are known, in fact, they all undergo the same movement. A mean value for the optical flow can be expressed by using all the tracked features as $O \bar{F}_{x}=\bar{V}_{O F_{\mathrm{x}}}+K_{x} \bar{V}_{O F_{\mathrm{z}}}+\bar{R}_{O F_{x}}$ and $O \bar{F}_{y}=\bar{V}_{O F_{\mathrm{y}}}+K_{y} \bar{V}_{O F_{\mathrm{z}}}+\bar{R}_{O F_{y}}$. The terms $O \bar{F}_{x}$ and $O \bar{F}_{y}$ are the optical flow means measured in the image coordinate system, the relative depth is expressed as $\bar{V}_{O F_{\mathrm{z}}}$, and $K_{x}, K_{y}$ are constant scale factors depending on the intrinsic camera parameters. The rotational optical flow terms $\bar{R}_{O F_{x}}$ and $\bar{R}_{O F_{y}}$ are compensated and the pseudo-speeds $\left(\bar{V}_{O F_{x}}, \bar{V}_{O F_{y}}, \bar{V}_{O F_{z}}\right)$ are deduced, by using a similar approach than the one presented in [10]. This method provides $-z \frac{\bar{V}_{O F_{x}}}{\alpha_{x}}=\dot{x},-z \frac{\bar{V}_{O F_{y}}}{\alpha_{y}}=\dot{y}$ and $z \bar{V}_{O F_{z}}=\dot{z}$, where we are considering that the camera and Quad-plane arrangement share the same movement, i.e., $\left(\dot{x}^{c}, \dot{y}^{c}, \dot{z}^{c}\right)=(\dot{x}, \dot{y}, \dot{z})$.

D. States computation when the road is out of the camera's field of view

In a real experimental application, the image of the road can be lost in certain moments. To address this problem, let's define a binary signal $s:[0, \infty) \rightarrow\{0,1\}$ as

$$
s(t):= \begin{cases}0 & \text { road not detected at time } t \\ 1 & \text { road detected at time } t\end{cases}
$$

which allows to switch between two different strategies for computing the vehicle's states. We define also the time event $T_{s 0}$ as the time when the binary signal switches from 1 to 0 , and $T_{s 1}$ the time event when the binary signal switches from 0 to 1 .

First, consider the desired heading angle computation. The equation (6) permits to define

$$
\psi_{s}(t)=s(t) \psi_{d}(t)+(1-s(t)) \psi_{I M U}\left(T_{s 0}\right)
$$

where $\psi_{d}(t)$ is obtained from (4), $\psi_{I M U}\left(T_{s 0}\right)$ is the IMU's heading angle measurement at $T_{s 0}$, and $\psi_{s}(t)$ is the desired heading angle that will be used in the Quad-plane embedded controller. From this result, (7) allows switching from a $\psi_{s}(t)$ generated by the imaging algorithm, and a $\psi_{s}(t)$ defined by the IMU's heading angle value at the time when the road was not detected on the image.

Now, lets consider the vehicle's lateral position estimation when the road is out of the camera's field of view. Equation (6) permits to estimate

$$
\begin{aligned}
e_{y s}(t) & =s(t) e_{y}(t) \\
& +(1-s(t))\left(e_{y}\left(T_{s 0}\right)+\int_{T_{s 0}}^{T_{s 1}} \dot{y}(t) d(t)\right)
\end{aligned}
$$

where $e_{y}(t)$ is obtained as in (5), $e_{y}\left(T_{s 0}\right)$ is the lateral position estimation at $T_{s 0}$, and $\dot{y}(t)$ is the vehicle's lateral velocity obtained from optical flow estimation. From the equation (8), it is possible to switch between two different methods for estimating the vehicle's a lateral position w.r.t. the road. In the first method, the lateral position is obtained from the road line detection in the camera's image. The second method uses the road's position at $T_{s 0}$ in combination with the integral of $\dot{y}$ during the time the road is not being detected by the imaging algorithm, i.e., during a time defined by time events $T_{s 0}$ and $T_{s 1}$.

\section{Control Strategy}

The proposed control strategy is based on the idea that the global system (1) is constituted of two subsystems, the attitude dynamics and the position dynamics, each one with a time-scale separation between them [11]. From this fact, it is possible to propose a hierarchical control scheme where the position controller outputs desired attitude angles $\left(\theta_{d}, \phi_{d}\right.$ and $\psi_{d}$ ) which are the angles to be tracked by the orientation controllers.

\section{A. Attitude Control}

The integral sliding mode control is used for stabilizing the attitude dynamics of the Quad-plane. The approach is explained for the pitch dynamics, but the same procedure must be followed for generating the roll and yaw dynamics. The error equation for the pitch sub-system is defined as $\tilde{\theta}_{1}=\theta_{1}-\theta_{1_{d}}$. Lets choose the switching function defined in [12] as

$$
\varphi(\theta, t)=\dot{\tilde{\theta}}_{1}+2 \lambda \tilde{\theta}_{1}+\lambda^{2} \int_{0}^{t} \tilde{\theta}_{1}(\tau) \mathrm{d} \tau
$$


In (9) the parameter $\lambda$ is the slope of the sliding line, which should accomplished $\lambda>0$ to ensure the asymptotic stability of the sliding mode. The time derivative of (9) can be calculated as $\dot{\varphi}=u_{2}+2 \lambda \theta_{2}+\lambda^{2} \tilde{\theta}_{1}$, and from the sliding mode condition $\dot{\varphi}=0$, we find the equivalent control $u_{2_{e q}}=-2 \lambda \theta_{2}-\lambda^{2} \tilde{\theta}_{1}$. For obtaining a control law such that $\tilde{\theta}_{1}$ remains on the sliding surface $\varphi(\theta, t)=0, \forall t>0$, we propose the Lyapunov function candidate $v(\varphi)=\frac{1}{2} \varphi^{2}$. A condition for the stability of the pitch sub-system is satisfied if we can ensure that the condition $\dot{v}(\varphi)=\frac{1}{2} \frac{d}{d t} \varphi^{2} \leq \eta|\varphi|$ holds for $\eta \geq 0$. Thus, the system remains on the sliding surface and the states go to the origin. Then $\varphi \dot{\varphi} \leq-\eta|\varphi|$ and the controller must be chosen in a way that $\theta_{1}=$ $u_{2_{e q}}-K \operatorname{sign}(\varphi)$ where $K>0$.

\section{B. Position Control}

The movement in the $x-y$ plane is generated by orientating the vehicle's thrust vector in the direction of the desired displacement. Then, $\theta_{d}$ and $\phi_{d}$ behaves as virtual controllers for the positioning dynamics. The control proposed for $z$ an $x$, respectively, are defined by

$$
\begin{aligned}
& u_{1}=k_{p z}\left(z_{1}-z_{1_{d}}\right)+k_{v z}\left(z_{2}-z_{2_{d}}\right)+1 \\
& \theta_{d}=\frac{k_{p x}\left(x_{1}-x_{1_{d}}\right)+k_{v x}\left(x_{2}-x_{2_{d}}\right)}{u_{1}}
\end{aligned}
$$

where $k_{v x}, k_{p x}, k_{p z}$ and $k_{v z}$ are positive real numbers.

1) y-Position Control: The lateral position control is formed by a pair of PID controllers, one for the case where the highway is detected and the other one for the case where it is not. The PID controllers have different gains with the goal of obtaining a behavior like a PD (when the highway is inside of the camera's field of view) and a PI (when the highway is not detected) controller according to the gain values chosen. The PD controller ensures a rapid response for maintaining the vehicle's $y$ coordinate at a minimum value, i. e., $y=0$ [5]. The PI controller is used when the vehicle looses the image of the highway, since at this moment a switch to an alternative method for measuring the $\psi$ angle and $y$ position occurs. In both cases the control objective is to regulate the $y_{1}$ state to the origin, i.e. $y_{1 d}=0$.

The control schema when the line is detected, proposes a feedback control law given by

$$
\phi_{1}=\frac{1}{u_{1}}\left(-k_{L 3} y_{1}-k_{L 4} y_{2}+k_{L I} \xi\right)
$$

where $k_{L 3}, k_{L 4}$ and $k_{L I}$ are positive real numbers. Here we have introduced the additional state $\xi$, where $\xi$ dynamics are given by $\dot{\xi}=y_{1 d}-y_{1}=-y_{1}$. Defining a distance named $d_{c}$ as the lateral position existing between the vehicle's center of gravity projection and the point where the camera loses the image of the highway (Fig. 3), a change of coordinates can be made such that $y_{d_{1}}=y_{1}+d_{c}$ and $\dot{y}_{d_{1}}=y_{d_{2}}=y_{2}$ is its derivative. Thus, using the control (12), the closed-loop system of the $y$ dynamics is given by

$$
\begin{aligned}
& \dot{y}_{d_{1}}=y_{d_{2}} \\
& \dot{y}_{d_{2}}=-k_{L 3} y_{d_{1}}-k_{L 4} y_{d_{2}}-k_{L I} \xi \\
& \dot{\xi}=-y_{d_{1}}
\end{aligned}
$$

with $e_{y_{d}}=\left(y_{d_{1}}, y_{d_{2}}, \xi\right)^{T}$. Then, (13) can be represented as $\dot{e}_{y_{d}}=A_{L} e_{y_{d}}$ where

$$
A_{L}=\left(\begin{array}{ccc}
0 & 1 & 0 \\
-k_{L 3} & -k_{L 4} & k_{L I} \\
-1 & 0 & 0
\end{array}\right)
$$

The control schema when the line is not detected proposes the same structure given by (12), with the difference of having a set of alternative gains. Thus, the closed-loop system is represented by $\dot{e}_{y_{d}}=A_{N L} e_{y_{d}}$, where

$$
A_{N L}=\left(\begin{array}{ccc}
0 & 1 & 0 \\
-k_{N L 3} & -k_{N L 4} & k_{N L I} \\
-1 & 0 & 0
\end{array}\right)
$$

here $k_{N L 3}, k_{N L 4}$ and $k_{N L I}$ are positive real numbers.

It is important to mention that in the experimental platform, the gains were chosen experimentally, this resulted in different values for both cases. When the road is detected, the values were selected as $K_{L 3}=1800, K_{L 4}=650$ and $K_{L I}=1$. Gains were selected as $k_{N L 3}=1800, k_{N L 4}=1$ and $k_{N L I}=20$ for the case where the highway is not detected.

\section{C. y-Position Control Stability Analysis}

In this section we prove the stability in each region as well as in switching boundaries, i.e., when the gains of (12) switch to alternative values.

It is possible to find a common Lyapunov function for the closed-loop system formed applying the two controllers of the lateral position dynamics [13]. However, under this approach, same pole locations need to be chosen in the two cases: when the highway is detected and when it is not. For the present application different gain values are being applied. Thus, we can define a state-dependent switched linear system given by the closed-loop system and the switching conditions

$$
\dot{e}_{y_{d}}=\left\{\begin{array}{cc}
A_{L} e_{y_{d}} & \text { if } y_{d_{1}}<0 \\
A_{N L} e_{y_{d}} & \text { if } y_{d_{1}} \geq 0
\end{array}\right.
$$

Note that each individual system in (16) is stable, since the matrices $A_{L}$ and $A_{N L}$ are Hurwitz.

Now, suppose that there exists a family $A_{p}, p \in \mathcal{P}$ of functions from $\Re^{n}$ to $\Re^{n}$, with $\mathcal{P}=1,2, \ldots, m$ defining the finite index set. For the linear system case, this results in a family of systems $\dot{x}=A_{p} x$ with $A_{p} \in \Re^{n \times n}$. Now we define a piecewise constant function $\sigma:[0, \infty) \rightarrow \mathcal{P}$ with finite number of discontinuities, named switching times, defined on every bounded time interval. Then $\sigma$ gives the index $\sigma(t) \in$ $\mathcal{P}$ of the system that is active at each instant of time $t$. The next theorem proves the stability of the whole system including in the times where the switch occurs:

Theorem 1: Consider vectors $t_{p q}$, symmetric matrices $S_{p}$ with $\Omega_{p} \in\left\{x: x^{T} S_{p} x \geq 0\right\}, \forall p \in \mathcal{P}$ having non-negative entries and symmetric matrices $P_{p}$ such that:

$$
\begin{array}{r}
A_{p}^{T} P_{p}+P_{p} A_{p}+\beta_{p} S_{p}<0, \quad \beta_{p} \geq 0 \\
0<P_{p}-P_{q}+f_{p q} t_{p q}^{T}+t_{p q} f_{p q}^{T} \text { for some } t_{p q} \in \Re^{n}
\end{array}
$$


With the boundary between $\Omega_{p}$ and $\Omega_{q}$ of the form $\{x$ : $\left.f_{p q}^{T}=0\right\}, f_{p q} \in \Re^{n}$. Then every continuous, piecewise $\mathcal{C}^{1}$ trajectory of the system $\dot{x}=A_{\sigma} x$ tends to zero exponentially.

Before proving Theorem 1, let's use the following theorem.

Theorem 2: The system $\dot{x}=f(t, x), f(t, x) \equiv 0$, is exponentially stable on the region $D=\left\{x \in \Re^{n} \mid\|x\|<r\right\}$ if there exists a Lyapunov function $V(t, x)$ and some positive constants $c_{1}, c_{2}, c_{3}$, such that $\forall(t, x) \in[0, \infty) \times D_{0}$, $D_{0}=\left\{x \in \Re^{n} \mid\|x\|<r / m\right\}$

$$
\begin{array}{r}
c_{1}\|x\|^{2} \leq V(t, x) \leq c_{2}\|x\|^{2} \\
\frac{\partial V}{\partial t}+\frac{\partial V}{\partial x} \leq-c_{3}\|x\|^{2}
\end{array}
$$

where $m$ is the overshot from definition of exponential stability.

Proof: See [14], pp. 169.

Proof: [Proof of Theorem 1 ] The proof relies on the Theorem 2, then using the Lyapunov function candidate $V(x)=x^{T} P_{p} x$ and assuming that $x(t)$ is continuous and piecewise $\mathcal{C}^{1}$, hence, $V(t)$ has the same characteristics. Premultiplying and postmultiplying the condition (18) by $x$, the inequality on the left side of (19) is satisfied. In the same way, inequality (20) follows if we premultiply and postmultiply both sides of (17) by $x$.

\section{EXPERIMENTAL PLATFORM}

\section{A. Quad-plane platform}

The control algorithm is implemented on the the Gumstix computer that is based on a $720 \mathrm{MHz}$ ARM Cortex-A8 CPU with 512MB Flash memory. It is the heart of the embedded system and constitutes the Flight Control Computer (FCC). The control law is executed in real-time at a $100 \mathrm{~Hz}$ frequency. Inertial measurements are provided at $100 \mathrm{~Hz}$ by means of a 3DMGX3-25 Inertial Measurement Unit (IMU) from Microstrain ${ }^{\circledR}$. For more details of the experimental platform see [1].

\section{B. Embedded vision system}

The embedded vision system includes a PlayStation ${ }^{\circledR}$ Eye camera, and a Flying Netbook computer, where the vision algorithms are programmed. The camera is capable of providing 120 images per second of a $320 \times 240$ resolution. The camera is used for observing the scene below the vehicle and record the image and video. The translational velocity in the $x-y$ plane is obtained from an optical flow algorithm, which is based on the pyramidal Lucas-Kanade method.

\section{Ground station}

The helicopter is communicated wireless to a ground station PC where a graphical user interface (GUI) allows monitoring and controlling the Quad-plane. The user can visualize, in real-time, graphics representing the measurements from the on-board sensors as well as graphics representing the control law computation.
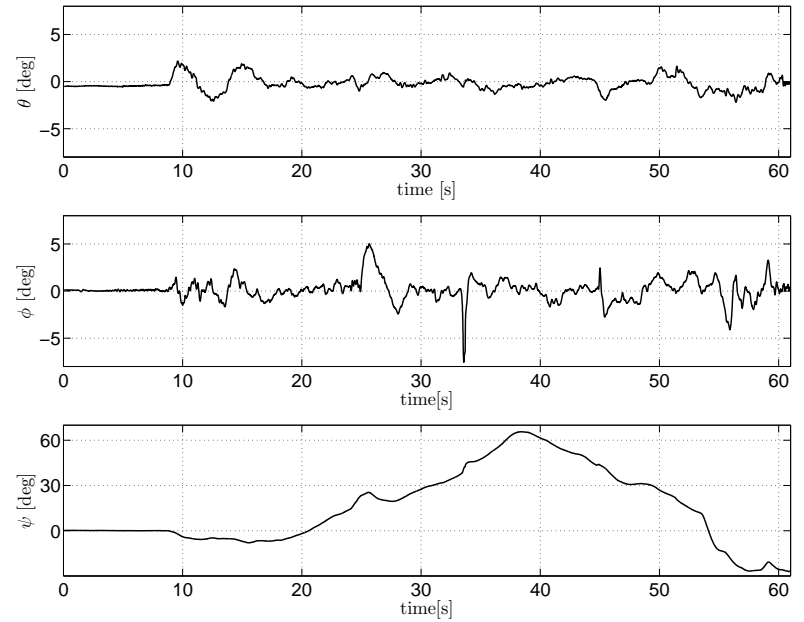

Fig. 4. Behavior of the Euler angles during the experiment. The system was disturbed in the $\psi$ angle at $t=34 \mathrm{~s}$.

\section{EXPERIMENTAL RESULTS}

The strategies for states estimation and control proposed in Section IV and V, were validated in real-time experiments, which can be described as follows. An autonomous take-off is performed, where the goal is to achieve a desired altitude of $0.70 \mathrm{~m}$ over the road. Once at this height, the helicopter can detect the road, which allows orientating its heading and stabilizing its lateral position, achieving a flying path well centered over the road. The vehicle is then required to navigate forward at a desired speed of $1 \mathrm{~m} / \mathrm{s}$, regulating its heading angle and lateral position w.r.t. the road. Once the vehicle is near to the end of the road segment, the landing is performed autonomously, and the vehicle descends slowly to the ground.

The switching between the two states estimation approaches is illustrated in figure 7. The graphic on the middle represents the time instants where the line is being detected $(s=1)$ and where the line is not being detected $(s=0)$. The upper graphic represents the desired heading angle as computed from (7). The lower graphic represents the lateral position error as computed from (8). As we can see in the figures 4, 5, 6, 7 and 8, the vehicle was perturbed in $\psi$ angle and in the $y$ position. It is important to say that the graphs showed corresponds at the experiment that can be seen at http://www.youtube.com/watch?v=fAQvSXPAG5Q

\section{CONCLUDING REMARKS}

One limitation that we have seen in the real-time experiment was related to the maximum velocity in which the Quad-Plane can estimate and track the road. Due to the limitation on time of the vision algorithm the vehicle has a limited speed.

The zeno behavior was no studied in the theoretical part of the work, but in practice, this phenomena does not occur in real-time experiments. However, a study related on this phenomena should be treated on future works. 

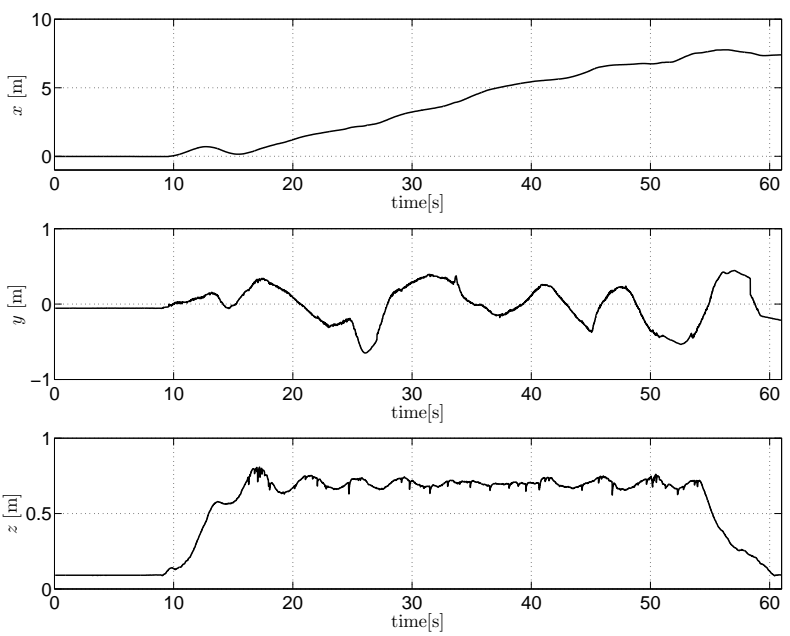

Fig. 5. Position of the vehicle during the experiment. The system was disturbed in the $y$ position at $t=26 \mathrm{~s}$ and $t=45 \mathrm{~s}$.
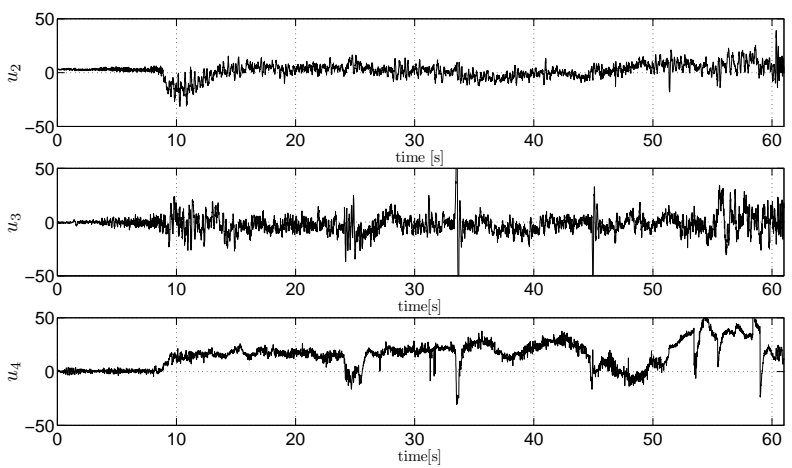

Fig. 6. Control inputs $u_{2}, u_{3}$ and $u_{4}$ of (1).
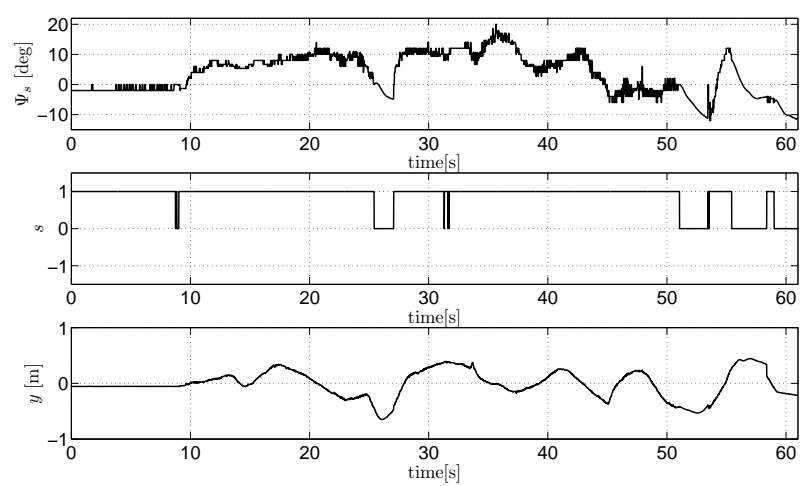

Fig. 7. Switching signal $s, \psi_{s}$ and $e_{y s}$. At $t=25$ and $t=51$ the vehicle was disturbed as we can see from the angle measurement $\psi_{d}$.

Future work will focus on the development of a hybrid control for switching not only controller gains but also control strategies.

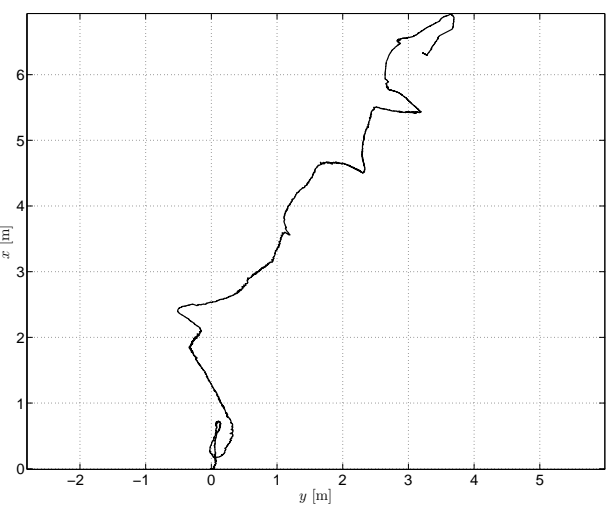

Fig. 8. $x-y$ plane of the navigated path. In this figure we show the switching effect in the lateral position dynamics (see peaks in $(x, y)=$ $(2.4,-0.55),(x, y)=(4.6,2.2)$ and $(x, y)=(5.45,3.15))$ when the system is disturbed and the camera losses the highway image.

\section{REFERENCES}

[1] G. Flores, J. Escareno, R. Lozano., and S.Salazar, "Quad-tilting rotor convertible mav: Modeling and real-time hover flight control," Journal of Intelligent and Robotic Systems, DOI: 10.1007/s10846-011-9589-x.

[2] L. G. Carrillo, E. Rondon, A. Sanchez, A. Dzul, and R. Lozano, "Stabilization and trajectory tracking of a quad rotor using vision," Journal of Intelligent and Robotic Systems, vol. 61, no. 1-4, pp. 103118, January, 2011.

[3] E. Rondon, L. Garca-Carrillo, and I. Fantoni, "Vision-based altitude, position and speed regulation of a quad-rotor rotorcraft," in International Conference on Intelligent Robots and Systems, Taipei, Taiwan, October, 2010, pp. $628-633$.

[4] J. Hespanha, O. Yakimenko, I. Kaminer, and A. Pascoal, "Linear parametrically varying systems with brief instabilities: An application to integrated vision/imu navigation," IEEE Transactions on Aerospace and Electronic Systems, vol. 40, no. 3, pp. 889-902, July, 2004.

[5] G. Hoffmann, H. Huang, S. L. Waslander, and C. Tomlin, "Quadrotor helicopter flight dynamics and control: Theory and experiment," in Proc. of the AIAA Guidance, Navigation, and Control Conference, Hilton Head, South Carolina, USA, 2007.

[6] M. Oishi and C. Tomlin, "Switching in nonminimum phase systems: Application to vstol aircraft," in Proc. IEEE American Control Conference (ACC'2000), Chicago, IL, Jun. 2000, pp. 838-843.

[7] S. Bouabdallah and R. Siegwart, "Backstepping and sliding-mode techniques applied to an indoor micro quadrotor," in In Proceedings of IEEE Int. Conf. on Robotics and Automation, Barcelona, Spain, Apr. 2005, pp. 2247-2252.

[8] F. Chaumette, "Image moments: a general and useful set of features for visual servoing," IEEE Trans. on Robotics, vol. 20, no. 4, pp. 713-723, August, 2004.

[9] G. Bradski and A. Kaehler, Learning OpenCV: Computer Vision with the OpenCV Library. United States of America: O'Reilly Media, 2008

[10] E. Rondon, I. Fantoni-Coichot, A. Sanchez, and G. Sanahuja, "Optical flow-based controller for reactive and relative navigation dedicated to a four rotor rotorcraft," in IEEE/RSJ International Conference on Intelligent Robots and Systems, St. Louis, USA, October, 2009, pp. 684-689.

[11] P. Kokotović, H. K. Khalil, and J. O'Reilly, Singular Perturbation Methods in Control: Analysis and Design. London: Academic Press: Siam, 1999.

[12] J. Slotine and W. Li, Applied Nonlinear Control. Prentice Hall, 1990.

[13] D. Liberzon, Switching in Systems and Control. Boston: Birkhuser, 2003.

[14] H. K. Khalil, Nonlinear Systems. New York: Prentice Hall, 2002. 